\title{
Pré-excitação Ventricular como Causa de Disfunção Ventricular Esquerda Parcialmente Reversível com Ablação da via Anômala
}

\author{
Ventricular Pre-excitation Causing Left Ventricular Dysfunction Partially \\ Reverted After Ablation of the Accessory Pathway \\ João Paulo Chaves de Melo1', Maria Licia Ribeiro Cury Pavão1', Elerson Arfelli', Marcelo Garcia Leal', \\ José Antonio Marin-Neto ${ }^{1}$, Andre Schmidt ${ }^{1, *}$
}

ORCID IDS

Melo JPC (D) https://orcid.org/0000-0001-9301-0977

Pavão MLRC (D) https://orcid.org/0000-0002-6963-3776

Arfelli E (D) https://orcid.org/0000-0002-8085-2195

Leal MG (D) https://orcid.org/0000-0002-5458-1782

Marin-Neto JA (D) https://orcid.org/0000-0002-8651-8833

Schmidt A (D) https://orcid.org/0000-0002-1090-8165

\section{RESUMO}

A pré-excitação ventricular é causa rara de cardiomiopatia induzida ou mediada por arritmias. As vias acessórias à direita, especificamente com padrão de bloqueio de ramo esquerdo, podem causar disfunção ventricular pela ativação ventricular anormal resultante da condução anterógrada pela via acessória, por causar dessincronismo atrioventricular, interventricular e intraventricular, com contração assíncrona da parede ventricular e regurgitação mitral. Foi descrita uma paciente assintomática, com eletrocardiograma exibindo pré-excitação ventricular, bloqueio do ramo esquerdo e disfunção ventricular sistólica moderada. Estudo eletrofisiológico demonstrou via acessória de localização anterior e com período refratário anterógrado de 600 ms, realizando-se ablação por radiofrequência com sucesso e significativa melhora da função ventricular.

PALAVRAS-CHAVE: Disfunção ventricular; Síndrome de préexcitação; Ablação por cateter.

\begin{abstract}
Ventricular pre-excitation is one of the rarest causes of cardiomyopathy induced or mediated by arrhythmia. Right accessory pathways, specifically with left bundle branch block pattern, can cause ventricular dysfunction, since abnormal ventricular activation resulting from anterograde atrioventricular conduction can cause atrioventricular, interventricular, and intraventricular dyssynchrony, with asynchronous contraction of the ventricular wall and mitral regurgitation. An asymptomatic patient, with ventricular pre-excitation with left bundle branch block and moderate ventricular dysfunction at echocardiography was described. The electrophysiological study demonstrated an accessory route of anterior location and with an anterograde refractory period of $600 \mathrm{~ms}$, successfully performing radiofrequency ablation and substantial improvement of ventricular function.
\end{abstract}

KEYWORDS: Ventricular dysfunction; Wolff-Parkinson-White syndrome; Catheter ablation.

1. Universidade de São Paulo - Faculdade de Medicina de Ribeirão Preto - Hospital das Clínicas - Ribeirão Preto (SP) - Brasil.

*Autor correspondente: aschmidt@fmrp.usp.br

Recebido: 08 Nov 2019 | Aceito: 19 Nov 2019

Editor Associado: J. Tarcísio Medeiros de Vasconcelos 


\section{INTRODUÇÃO}

As vias anômalas acessórias são tecidos remanescentes resultantes do incompleto desenvolvimento embriológico do anel atrioventricular (AV) e da falha da separação fibrosa entre os átrios e os ventrículos. $\mathrm{Na}$ síndrome de pré-excitação ventricular, também conhecida como síndrome de Wolff-Parkinson-White, a condução AV ocorre, parcial ou totalmente, por meio de via acessória, que resulta em ativação mais precoce (pré-excitação) dos ventrículos ${ }^{1}$.É uma anomalia relativamente comum, com estimativa de acometer 1-3/1000 nascidos vivos ${ }^{1}$. Pode ser assintomática ou manifestar-se como taquicardia paroxística supraventricular (TPSV) ou ainda embora mais raramente se apresentar como síncope ou morte súbita devido à rápida condução de uma taquiarritmia atrial pela via acessória ${ }^{2}$. Apesar de episódios ocasionais de TPSV geralmente não estarem associados ao desenvolvimento de disfunção ventricular, existe essa possibilidade em casos de taquicardia incessante. Adicionalmente, a ativação ventricular anormal resultante da condução precoce anterógrada pode causar dessincronia atrioventricular, interventricular e intraventricular e também resultar em cardiomiopatia ${ }^{3}$.

Neste relato de caso apresenta-se paciente com pré-excitação do ventrículo direito e função ventricular esquerda deprimida, em que a ablação bem sucedida da via acessória resultou em melhora substancial da fração de ejeção do ventrículo esquerdo (FEVE).

\section{RELATO DE CASO}

D.A.B., 39 anos, sexo feminino, portadora da síndrome de imunodeficiência humana e hepatite $\mathrm{C}$, em acompanhamento ambulatorial, mantendo carga viral indetectável. Assintomática do ponto de vista cardiovascular, iniciou avaliação pré-tratamento para hepatite $\mathrm{C}$, sendo identificado ao eletrocardiograma (ECG) um intervalo PR curto e onda delta típica. O ECG sugeria padrão de bloqueio do ramo esquerdo (BRE), compatível com via acessória de localização em anel tricuspídeo (Fig. 1). O ecocardiograma transtorácico, realizado em janeiro de 2016, revelou dilatação do ventrículo esquerdo (VE), com diâmetro telediastólico (DDF) de $58 \mathrm{~mm}$ e disfunção ventricular esquerda moderada (FEVE 36\%). Não havia história familiar de cardiomiopatia, nenhuma doença viral no passado recente, sendo também descartado coronariopatia por cintilografia miocárdica de perfusão em esforço e repouso.

Realizada estratificação de risco associado à via acessória com Holter de 24 horas evidenciando préexcitação durante todo o registro e teste ergométrico, sem perda súbita do padrão de pré-excitação ventricular. A seguir, foi realizado estudo eletrofisiológico (junho de 2016), que mostrou intervalos AH: 28 ms e HV: $4 \mathrm{~ms}$, havendo uma via acessória com propriedades de condução anterógrada e retrógrada e com período refratário efetivo anterógrado de $600 \mathrm{~ms}$, sugerindo baixo risco para eventos cardíacos arrítmicos graves. Durante mapeamento endocárdico, foi observada fusão AV em região anterior do anel tricúspideo, sendo aplicados pulsos de radiofrequência que levaram a desaparecimento das conduções anterógrada e retrógrada pela via acessória. Repetido ecocardiograma em setembro de 2016, que evidenciou nítida melhora da FEVE (46\%), do DDF: $52 \mathrm{~mm}$ e também ECG sem evidência de pré-excitação ventricular (Fig. 2). Em paralelo, foi iniciado tratamento

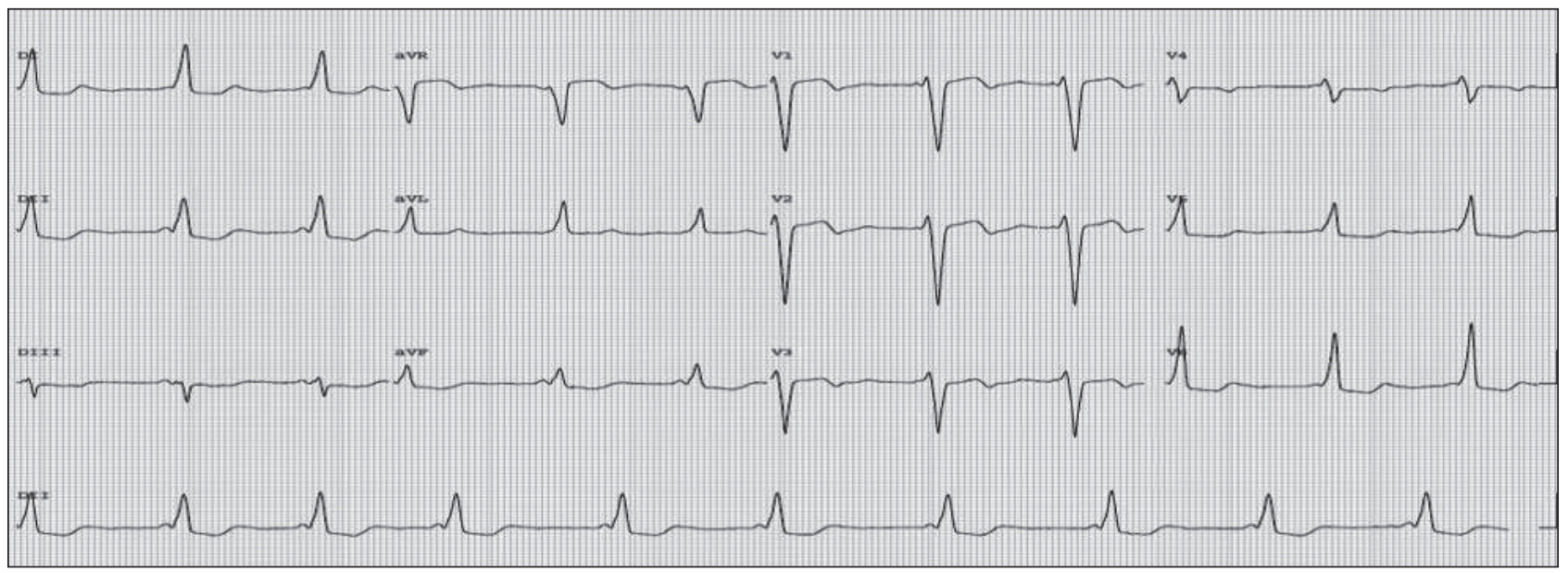

Figura 1. Eletrocardiograma de 12 derivações pré ablação. 


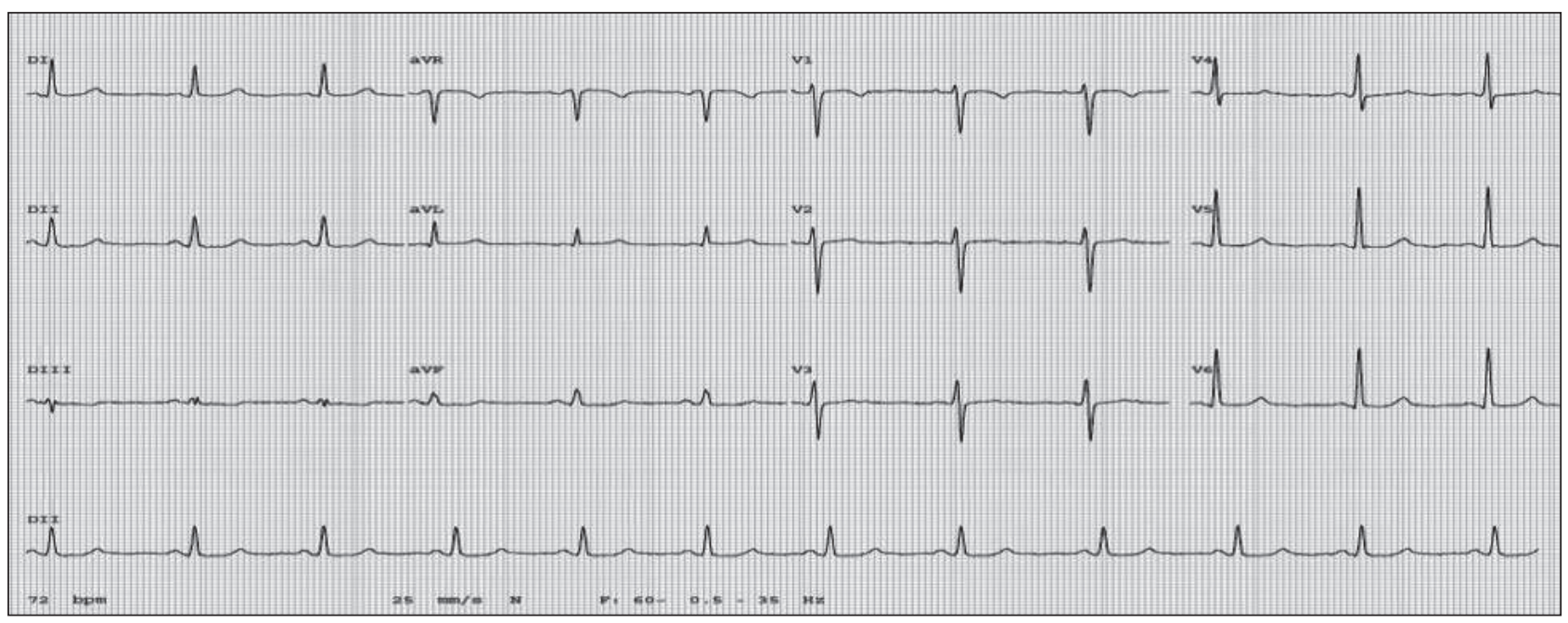

Figura 2. Eletrocardiograma de 12 derivações após ablação

com sofusbuvir e daclatavir em fevereiro de 2017 por 12 semanas com negativação da carga viral.

Mantém-se atualmente assintomática em seguimento ambulatorial, com função ventricular sistólica estável no limite inferior da normalidade, em uso extremamente irregular de betabloqueador (carvedilol) e inibidor da enzima conversora da angiotensina, medicações iniciadas após a ablação.

\section{DISCUSSÃO}

Neste relato de caso, pode-se plausivelmente concluir que a ablação de via acessória conseguiu praticamente reverter uma cardiomiopatia induzida por dessincronismo ventricular secundária a via acessória de padrão de BRE.

Sabidamente, o BRE provoca movimento assíncrono da parede ventricular, com potencial para evoluir com disfunção ventricular e regurgitação mitral ${ }^{4} \mathrm{e}$ foi demonstrado que, em pacientes sem outra causa evidente de disfunção sistólica ventricular, a "eliminação" efetiva do padrão do BRE e dessincronia intraventricular dele resultante, é a base para a melhora funcional do $\mathrm{VE}^{5}$. Reforçando o papel da dessincronia na patogênese da insuficiência cardíaca (IC), diversos estudos avaliaram o impacto clínico da terapia de ressincronização cardíaca na reversão da disfunção ventricular ${ }^{6,7}$.

Além disso, tornou-se amplamente aceito com base em ensaios clínicos relativamente recentes que a estimulação do ventrículo direito (VD) a longo prazo promove a progressão da insuficiência cardíaca devido à deterioração da função do
VE. Novamente, parece provável que a estimulação do VD resulte em cenário essencialmente equivalente ao dessincronismo ventricular induzido por BRE, com efeito adverso na estrutura e funcionalidade do $\mathrm{VE}^{8}$.

Finalmente, há na literatura vários relatos descrevendo pacientes com extrassístoles ventriculares $(\mathrm{EV})$ frequentes (> 20\%) originando-se da via ejetiva do $\mathrm{VD}$, com dilatação do VE e redução da FEVE. A ablação com sucesso por cateter reverteu a dilatação e disfunção do VE. Presume-se que as $\mathrm{EV}$ que manifestam morfologia de BRE deram origem a dessincronia e disfunção do $\mathrm{VE}^{9}$.

No caso de pré-excitação ventricular, a disfunção ventricular na ausência de taquicardia sustentada é manifestação menos comum, com apenas relatos de casos isolados. A maioria dos casos relatados relacionados a dessincronismo ventricular estava associada a uma via acessória póstero-septal direita. No entanto, vias acessórias anterior direita, lateral direita e vias fascículoventriculares também foram implicadas ${ }^{10}$. $\mathrm{O}$ tratamento com ablação por radiofrequência reverte a disfunção ventricular na maioria dos casos, pois elimina-se a dessincronia ventricular ${ }^{11}$.

\section{CONCLUSÃO}

Dados os relatos anteriores sobre o impacto potencial do padrão de ativação tipo BRE na dessincronia e na função ventricular, bem como a exclusão de causas mais comuns de disfunção ventricular, é plausível admitir-se que a pré-excitação do ventrículo direito tenha causado 
dilatação reversível e disfunção ventricular esquerda. Assim, em pacientes com via acessória manifesta em anel tricuspídeo e função cardíaca deprimida, a ablação por cateter para eliminar a pré-excitação pode ter efeitos benéficos sobre a função cardíaca, além de eliminar sua suscetibilidade a taquiarritmias recorrentes.

\section{CONTRIBUIÇÃO DOS AUTORES}

\author{
Conceitualização,Melo JPC e Pavão MLR; Metodologia,
} Melo JPC; Investigação, Arfelli E; Pavão MLRC e Leal MG; Redação - Primeira versão, Melo JPC; Redação - Revisão \& Edição, Schmidt A e Marin-Neto JA; Supervisão, Schmidt A.

\section{REFERÊNCIAS}

1. Cohen MI, Triedman JK, Cannon BC, Davis AM, Drago F, Janousek J, et al. PACES/HRS Expert Consensus Statement on the Management of the Asymptomatic Young Patient with a Wolff-Parkinson-White (WPW, Ventricular Preexcitation) Electrocardiographic Pattern: Developed in partnership between the Pediatric and Congenital Electrophysiology Society (PACES) and the Heart Rhythm Society (HRS). Endorsed by the governing bodies of PACES, HRS, the American College of Cardiology Foundation (ACCF), the American Heart Association (AHA), the American Academy of Pediatrics (AAP), and the Canadian Heart Rhythm Society (CHRS). Heart Rhythm. 2012;9:1006-24. https://doi. org/10.1016/j.hrthm.2012.03.050

2. Kohli U, Pumphrey $\mathrm{KL}$, Ahmed A, Das S. Pre-excitation induced ventricular dysfunction and successful berlin heart explantation after accessory pathway ablation. J Electrocardiol. 2018;51(6):1067-70. https://doi.org/10.1016/j. jelectrocard.2018.09.008

3. Shan Q, Jin Y, Cao K. Reversible left ventricular dyssynchrony and dysfunction resulting from right ventricular preexcitation. EP Europace. 2007;9(8):697-701. https://doi. org/10.1093/europace/eum138

4. Bradley DJ, Bradley EA, Baughman KL, Berger RD, Calkins $\mathrm{H}$, Goodman SN, et al. Cardiac resynchronization and death from progressive heart failure: a meta-analysis of randomized controlled trials. JAMA. 2003;289(6):730-40. https://doi.org/10.1001/jama.289.6.730

5. Blanc J, Fatemi M, Bertault V, Baraket F, Etienne Y. Evaluation of left bundle branch block as a reversible cause of nonischaemic dilated cardiomyopathy with severe heart failure. A new concept of left ventricular dyssynchrony-induced cardiomyopathy. EP Europace. 2005;7(6):604-10. https:// doi.org/10.1016/j.eupc.2005.06.005
6. William TA, David LH. Cardiac resynchronization therapy for heart failure. Circulation. 2003;108(21):2596-603. https:// doi.org/10.1161/01.CIR.0000096580.26969.9A

7. William TA, Fisher WG, Smith AL, Delurgio DB, Leon AR, Loh $E$, et al. Cardiac resynchronization in chronic heart failure. N Engl J Med. 2002;346(24):1845-53. https://doi.org/10.1056/ NEJMoa013168

8. Tops LF, Schalij MJ, Holman ER, Van Erven L, Van Der Wall EE, Bax J. Right ventricular pacing can induce ventricular dyssynchrony in patients with atrial fibrillation after atrioventricular node ablation. J Am Coll Cardiol. 2006;48(8):1642-8. https://doi.org/10.1016/j. jacc.2006.05.072

9. Takemoto M, Yoshimura H, Ohba Y, Matsumoto Y, Yamamoto $U$, Mohri $M$, et al. Radiofrequency catheter ablation of premature ventricular complexes from right ventricular outflow tract improves left ventricular dilation and clinical status in patients without structural heart disease. J Am Coll Cardiol. 2005;45(8):1259-1265. https://doi.org/10.1016/j. jacc.2004.12.073

10. Tomaske M, Janousek J, Razek V, Gebauer RA, Tomek V, Hindricks $G$, et al. Adverse effects of Wolff-Parkinson-White syndrome with right septal or posteroseptal accessory pathways on cardiac function. EP Europace. 2008;10(2):181189. https://doi.org/10.1093/europace/eun005

11. Guo B, Dai C, Li Q, Li W, Han L. Hazards of ventricular pre-excitation to left ventricular systolic function and ventricular wall motion in children: analysis of 25 cases. Cardiol Young. 2019;29(3):380-388. https://doi.org/10.1017/ S1047951118002500 